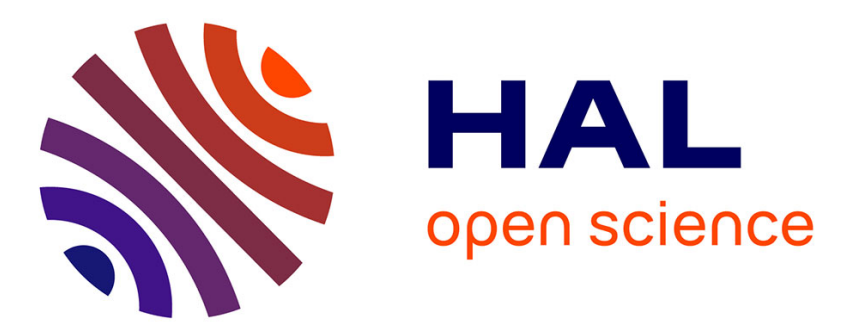

\title{
Electronion collision rates in atomic clusters irradiated by femtosecond laser pulses
}

\author{
M Moll, P Hilse, M Schlanges, Th Bornath, V P Krainov
}

\section{To cite this version:}

M Moll, P Hilse, M Schlanges, Th Bornath, V P Krainov. Electronion collision rates in atomic clusters irradiated by femtosecond laser pulses. Journal of Physics B: Atomic, Molecular and Optical Physics, 2010, 43 (13), pp.135103. 10.1088/0953-4075/43/13/135103 . hal-00569805

\section{HAL Id: hal-00569805 https://hal.science/hal-00569805}

Submitted on 25 Feb 2011

HAL is a multi-disciplinary open access archive for the deposit and dissemination of scientific research documents, whether they are published or not. The documents may come from teaching and research institutions in France or abroad, or from public or private research centers.
L'archive ouverte pluridisciplinaire HAL, est destinée au dépôt et à la diffusion de documents scientifiques de niveau recherche, publiés ou non, émanant des établissements d'enseignement et de recherche français ou étrangers, des laboratoires publics ou privés. 


\title{
Electron-ion collision rates in atomic clusters irradiated by femtosecond laser pulses
}

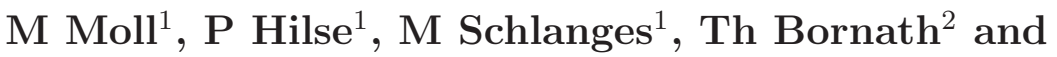 \\ V P Krainov ${ }^{3}$ \\ ${ }^{1}$ Institut für Physik, Ernst-Moritz-Arndt-Universität Greifswald, D-17487 \\ Greifswald, Germany \\ 2 Institut für Physik, Universität Rostock, D-18051 Rostock, Germany \\ ${ }^{3}$ Moscow Institute for Physics and Technology, 141700 Dolgoprudny, Moscow \\ Region, Russia \\ E-mail: moll@physik.uni-greifswald.de
}

\begin{abstract}
In atomic clusters irradiated by femtosecond laser pulses, plasmas with high density and high temperature are created. The heating is mainly caused by inverse Bremsstrahlung, i.e., determined by electron-ion collisions. In the description of the scattering of electrons on noble gas ions in such plasmas, it is important to account for the inner structure of the ions and the screening by the surrounding plasma medium which can be accomplished by using suited model potentials.

In a wide parameter range met in experiments, the Born approximation is not applicable. Instead, the electron-ion collision frequency is calculated on the basis of classical momentum transport cross sections. Results are presented for xenon, krypton, and argon ions in different charge states. A comparison of these results to those for the scattering on Coulomb particles with the same charge shows an enhancement of the collision frequency. The Born approximation, however, leads to an overestimation.
\end{abstract}




\section{Introduction}

In atomic clusters irradiated by femtosecond laser pulses, plasmas with high density and high temperature are created. Many experiments are performed using noble gas clusters interacting with lasers in the infrared regime with typical photon energies of 1.5 $\mathrm{eV}$. The initial plasma in the cluster is created due to tunnel ionization. The plasma will be heated by collisional absorption. This heating is accompanied by the second important ionization process, electron impact ionization. The heating of the plasma leads to a high pressure in the plasma and consequently to an expansion of the system. The average charge multiplicity of atomic ions inside the irradiated atomic clusters depends strongly on the laser intensity and pulse duration. It increases both with laser intensity and with its pulse duration.

For laser intensities of the order of $10^{15}-10^{16} \mathrm{~W} / \mathrm{cm}^{2}$, the first ionization step is tunneling ionization of atoms inside the cluster. The free electrons produced inside the cluster are quickly heated in the process of induced inverse bremsstrahlung (for infrared and visible laser light). These heated electrons collide with atomic ions and produce multiply charged atomic ions. This is the main mechanism for the production of highly charged ions. In laser pulses with a duration of 100-200 fs, charge multiplicities of 12 to 18 were found, see, for example, [1]. Petrov and Davis [2] showed for Xe clusters with 6000 atoms that at the pulse duration of $125 \mathrm{fs}$, wavelength of $800 \mathrm{~nm}$ and laser intensity of $10^{16} \mathrm{~W} / \mathrm{cm}^{2}$, the mean charge multiplicity of atomic ions is $\mathrm{Z}=14$. The mean electron energy during the laser pulse was found to be several keV.

For pulse durations of 20 to $40 \mathrm{fs}$, the electron heating is weaker and the maximum charge multiplicity of atomic ions is smaller. Last and Jortner [3] found for the same laser intensity of $10^{16} \mathrm{~W} / \mathrm{cm}^{2}$, but shorter laser pulse duration of $25 \mathrm{fs}$ in the case of Xe clusters with 1000 atoms, that the mean electron energy inside the cluster is only $50 \mathrm{eV}$. In this case, the mean charge multiplicity of atomic ions is $Z=8$. It should be noted also that the mean electron energy increases with the cluster size (see, for example, Fig. 2 in [4]).

In general, the energy of an electron is of the order of its ponderomotive energy (see, for example, Fig. 2 in Ref. [5]), which increases strongly with the field strength. When the laser intensity increases up to $6 \times 10^{16} \mathrm{~W} / \mathrm{cm}^{2}$, strong multiply ionization in Ar clusters with the charge multiplicity up to $\mathrm{Z}=16$ was found experimentally even in short laser pulses with the duration of $60 \mathrm{fs}$ [6].

For longer pulses of the order of 100-200 fs, the Mie resonance can be achieved during the laser pulse (typically on the rear side) as the cluster expands. In this resonance, the electric field inside the cluster increases strongly, leading to stronger heating and an additional multiply ionization of atomic ions. For example, the charge multiplicity of Ar ions increases on the rear side of the laser pulse up to $\mathrm{Z}=10$ at the

pulse duration of $150 \mathrm{fs}$, wavelength of $800 \mathrm{~nm}$, and laser intensity of $10^{16} \mathrm{~W} / \mathrm{cm}^{2}$ (see Fig. 2 in [7]). The role of the Mie resonance depends strongly on its width and on the temporal interval when the resonance condition is fulfilled. 
The aim of the present paper is not to consider the whole evolution of such a cluster. We want to focus on the important problem of the ponderomotive heating of the cluster, i.e., the heating due to the acceleration of electrons in the presence of multiply charged atomic ions inside the cluster [8, 9]. Besides the long range Coulomb part, the scattering of electrons on such ions can be influenced by the ions' inner structure. Our goal is to obtain realistic cross sections for elastic electron-ion scattering inside the noble gas clusters which can be used for further investigation of the cluster-laser dynamics. The present paper is organized as follows. After giving a short description of collisional heating in a laser field in Sec. 2, we discuss in Sec. 3 the problem of electronion scattering potentials accounting for the inner structure of the ions. In Sec. 4, the momentum transport cross section is considered. Numerical results for the scattering of electrons with noble gas atomic ions, namely the electron-ion transport cross section and the collision frequency, are presented in Sec. 5. Finally we draw some conclusions.

\section{Electron heating}

One important process in the interaction of intense femtosecond laser pulses with atomic clusters is the ponderomotive heating of the cluster. This heating is due to the acceleration of electrons in the presence of multiply charged atomic ions inside the cluster $[8,9]$. During the collisions of electrons with the ions, an electron can both absorb and emit laser photons. Because the probability of absorption is a little larger than the probability of induced emission [10], the electrons extract electromagnetic energy from the laser field which leads to a heating of the generated nanoplasma.

In the high-frequency limit, the energy absorbed by an electron in the time average (with respect to a cycle of the laser field) is

$$
\frac{\mathrm{d} E}{\mathrm{~d} t}=\nu_{e i} \Delta E_{e} .
$$

Here, the average energy, $\Delta E_{e}$, which an electron acquires during one collision with an atomic ion is twice the ponderomotive energy, namely

$$
\Delta E_{e}=\frac{e^{2} F(t)^{2}}{2 m_{e} \omega^{2}}
$$

where $\omega$ is the laser frequency and $F(t)$ is the electric field strength pulse envelope of the linearly polarized laser radiation inside the cluster. The electron-ion collision frequency $\nu_{e i}$ is given as

$$
\nu_{e i}=n_{i}\left\langle v \sigma_{\mathrm{tr}}(v)\right\rangle \approx n_{i} v_{e} \sigma_{\mathrm{tr}}\left(v_{e}\right),
$$

with $n_{i}$ being the number density of atomic ions inside the cluster and $\sigma_{\operatorname{tr}}$ the momentum transport cross section for elastic electron-ion scattering. The brackets denote averaging with respect to the electron velocity distribution which is approximated here by taking the value at the mean electron velocity $v_{e}$. Equation (1) together with expression (2) is valid under the condition $\omega>\nu_{e i}$, when the time between two successive collisions is longer than the period of laser field oscillations. For a general disscussion, see [8]. 
The simplest potential to be used for the description of electron scattering on free atomic ions is the Coulomb potential $V(r)=-Z / r$, where $\mathrm{Z}$ is the ion charge number. Here and throughout the following sections, Hartree atomic units, $e=m_{e}=\hbar=1$, are used. The screening due to the plasma electrons can be accounted for by the Debye potential,

$$
V_{D}(r)=-\frac{Z}{r} \exp (-\kappa r)
$$

where $\kappa=1 / r_{D}$ is the inverse of the Debye length $r_{D}=\left[k_{B} T_{e} /\left(4 \pi n_{e}\right)\right]^{1 / 2}$. In several papers, various approaches were used to calculate the electron-ion collision frequency and the dynamic conductivity, respectively, for classical plasmas under different conditions $[11,12,13,14]$. Quantum approaches to collisional absorption in the presence of a laser field were used in $[15,16,17,18]$. For strong fields, the electron-ion interaction can be considered in a perturbative approach. The influence of strong collisions in the case of weak fields can be be accounted for within a T-matrix approach [19, 20].

In the case of atomic ions with a distinct inner structure, i.e., the ion nucleus with charge $Z_{\text {nucl }} \gg Z$ is surrounded by certain "electron shells" containing the bound core electrons, a model potential which is more sophisticated than the simple Debye potential seems to be adequate. The most typical one is the Herman-Skillman potential which is derived based on the energy structure of isolated atoms and free atomic ions. Additionally it should be taken into account that the energy structure of an ion inside the cluster modifies strongly due to the surrounding plasma medium. Therefore, in order to obtain transport cross sections and electron-ion collision rates, one has to consider model potentials of atomic ions inside the cluster which depend also on cluster-plasma parameters, namely the electron temperature and number density.

\section{Electron-ion potential accounting for the inner structure of the ions}

Describing the scattering of electrons with atomic ions, one has to account for the inner structure of the ions as well as for screening effects caused by the surrounding plasma medium. Herman and Skillman [21] derived a computer code to calculate energy eigenvalues and effective potentials for the realistic electron-ion interaction in the case of isolated ions.

An analytical expression to account for the inner structure of the ions was proposed by Santra and Greene [23]

$$
V_{S G}(r)=-\frac{Z+\left(Z_{\text {nucl }}-Z\right) \exp (-a r)}{r}
$$

where $Z$ is the ion charge and $Z_{\text {nucl }}$ the nuclear charge number of the ion. The factor $\exp (-a r)$ describing the screening of the nucleus by the core electrons has to be determined in such a way that the binding energy $E_{n l}$ of the valence electron [24] is reproduced solving the single-particle Schrödinger equation with the potential (5). 
An analytic expression agreeing much better with the numerical data from Herman and Skillman than the potential (5) was given by Rogers [22]

$$
V_{R}(r)=-\frac{1}{r}\left(Z+\sum_{n=1}^{n^{*}} N_{n} e^{-\alpha_{n} r}\right)
$$

where $N_{n}$ is the number of electrons in the $n$th occupied electron shell, and $n^{*}$ is the number of shells. The screening parameters $\alpha_{n}$ have been determined by comparison of the calculated energy eigenvalues with experimental data [22].

In order to account for the surrounding plasma medium, an additional Debye screening factor $\exp (-\kappa r)$ may be introduced

$$
V_{R}^{s c}(r)=-\frac{1}{r}\left(Z+\sum_{n=1}^{n^{*}} N_{n} e^{-\alpha_{n} r}\right) \exp (-\kappa r) .
$$

For the calculation of the Debye length, $r_{D}=\left[k_{B} T_{e} /\left(4 \pi n_{i} \bar{Z}\right)\right]^{1 / 2}$, the cluster density is required. We will consider here the stage where the cluster expansion is not important yet and will adopt the number densities $n_{i}$ of liquid noble gases as typical values. Further, for simplicity, the mean ion charge in the plasma $\bar{Z}$ will be replaced by the respective ion charge $Z$. If, instead of the electron temperature $T_{e}$, the mean kinetic energy of electrons $E=3 k_{B} T_{e} / 2$ is assumed to be given, the Debye length can be calculated from $r_{D}=\left[E /\left(6 \pi n_{i} Z\right)\right]^{1 / 2}$.

\section{Transport cross sections for electron-ion scattering}

Before proceeding further, we want to discuss the conditions for the applicability of some approximations. The quantum momentum transport cross section is defined by

$$
\sigma_{\mathrm{tr}}=\int \mathrm{d} \Omega \frac{\mathrm{d} \sigma}{\mathrm{d} \Omega}(1-\cos (\theta))
$$

In first Born approximation, the differential cross section is in atomic units given as

$$
\frac{\mathrm{d} \sigma}{\mathrm{d} \Omega}=\frac{1}{4 \pi^{2}}|V(\mathbf{q})|^{2}
$$

where $V(\mathbf{q})$ is the Fourier transform of the potential $V(\mathbf{r})$, we have $q=2 k \sin (\theta / 2)$ with $\theta$ being the scattering angle and $k^{2}=2 E$. For the quantum mechanical scattering problem, the condition for the applicability of the perturbation theory (i.e., the Born approximation) is $[25](|V| / E) k d \ll 1$ where $V$ is the electron-ion interaction, $E$ is the kinetic energy of the electrons, $k$ is the electron wave number, and $d$ is the interaction radius.

We want to discuss this condition for the Debye potential. With $V_{D} \sim Z / r_{D}$ and $k=\sqrt{2 E}$, this condition takes the form $E \gg 2 Z^{2}$. In turn the condition for applicability of classical electron-ion scattering is $\left(\left|V_{D}\right| / E\right) k r_{D}>1$, leading to $E<2 Z^{2}$. For xenon clusters with 6000 atoms interacting with a laser pulse of $10^{16} \mathrm{~W} / \mathrm{cm}^{2}$ and $125 \mathrm{fs}$ duration, an ion charge multiplicity $Z=14$ was found [2]. Hence, we have the condition $E<10 \mathrm{keV}$. According to Fig. 5e of Ref. [2], the mean electron energy during 
the laser pulse is less than $9 \mathrm{keV}$. Thus, we can apply the approach of classical scattering for this case.

In another typical example [3], for the same laser intensity but shorter laser pulse duration of 25 fs and slightly smaller Xe clusters with 1000 atoms, charge states Z = 8 were achieved. According to the condition, the classical approach for electron-ion scattering is applicable when $E<3.5 \mathrm{keV}$. However, the electron heating is weaker due to the shorter pulse duration leading to a much smaller mean energy of electrons inside the cluster of $50 \mathrm{eV}$ (see Fig. 10 of [3]). Outer electrons ejected from the cluster have higher energy of about of $500 \mathrm{eV}$, but, of course, only electrons inside the cluster are of interest here. Thus, the condition of applicability for classical scattering depends strongly on the duration of a laser pulse at fixed intensity which both determine the electron heating. Ions species with lower $\mathrm{Z}$ are transient and occur at the earlier stages for lower energies where the condition $E<2 Z^{2}$ might be fulfilled also.

As we want to apply the results to the interaction of atomic clusters with laser fields, the question arises under which conditions the laser field can significantly distort the classical trajectory of electrons scattered on atomic ions inside the cluster. The influence of a laser field with the field strength $F$ upon the classical electron motion in the Debye potential can be neglected when the electric force is less than the force produced by the Debye potential, i.e. (in atomic units): $F<Z / r_{D}^{2}$. In the case of a xenon cluster with charge state $\mathrm{Z}=12$, the number density of atomic ions adopted to be that of liquid xenon, $n_{i}=0.00239$ a.u., and the electron energy $E=1 \mathrm{keV}$, one obtains $r_{D}=8$ a.u. and therefore $F<0.18$ a.u. corresponding to a laser intensity $I \sim 10^{15} \mathrm{~W} / \mathrm{cm}^{2}$. We can conclude, that there is a rather large parameter range where a classical description of the electron-ion collisions neglecting the influence of the laser field is appropriate.
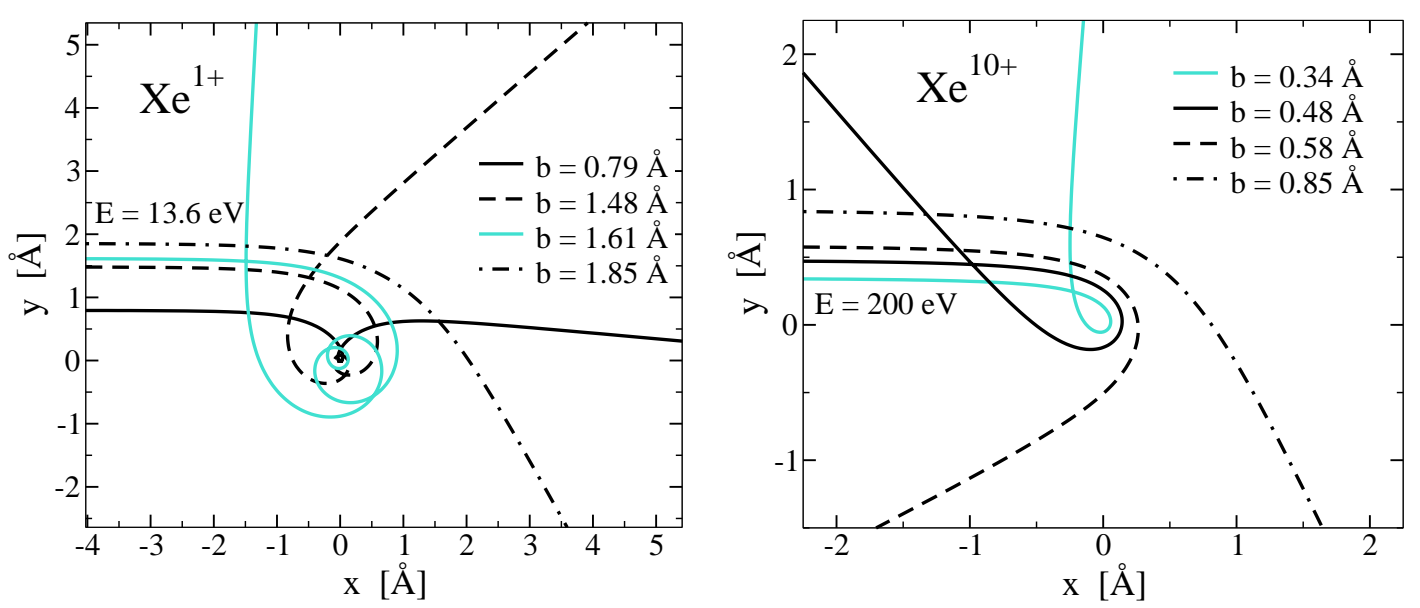

Figure 1. Electron trajectories for scattering on $\mathrm{Xe}^{1+}$ and $\mathrm{Xe}^{10+}$ ions with the screened model potential of Rogers (7). Electrons with kinetic energy $E=0.5$ a.u. and $E=7.4$ a.u., respectively, are approaching the ion in the center from the left side.

The momentum transport cross section $\sigma_{\text {tr }}$ for classical elastic scattering is given 
as

$$
\sigma_{\mathrm{tr}}=2 \pi \int_{0}^{b_{\max }} d b b(1-\cos \theta(b))
$$

where the scattering angle $\theta$ is derived from $[26]$

$$
\Theta(b)= \pm \theta(b)-2 \pi n, \quad \theta \in[0, \pi]
$$

via the deflection function

$$
\Theta(b)=\pi-2 \int_{r_{\min }}^{\infty} d r r^{-2}\left[b^{-2}\left(1-\frac{V(r)}{E}\right)-r^{-2}\right]^{-1 / 2} .
$$

Here, $b$ is the classical impact parameter and $r_{\min }$ is the classical distance of closest approach. In contrast to the desription of an isolated scattering event, the upper integration limit in eq. (10) has to be restricted to a finite value accounting for the

presence of neighboring ions. We have chosen in the calculations $b_{\max }=n_{i}^{-1 / 3}$. We remark that around this value, the results for the transport cross sections are not very sensitive to the choice of the precise value, e.g., half of this value for $b_{\max }$ would lead to a reduction by some percent.

Fig. 1 shows some typical electron trajectories for the scattering with ions via the model interaction potential $V_{R}^{s c}(7)$. The scattering angle depends on the impact parameter $b$. For large scattering parameters, the trajectories resemble those of the Coulomb case which are known to show no rotations. For smaller values of $b$ however, there can occur rotations of the trajectories in the case of the non-Coulombic model potential. Consequences of this behavior will be seen in the next section.

Having calculated the momentum transport section, we get finally the electronion collision frequency as an important quantity to describe the absorption of laser energy in the cluster given according to Eq. (3) where we use the mean electron velocity $v_{e}=\sqrt{2 E}=\sqrt{3 k_{B} T_{e}}$.

\section{Numerical results}

The collision frequency (3) determines the absorption of laser energy in the plasma. Because the dependence on the ion number density $n_{i}$ is just a factor, we consider first the product of the electron velocity and the transport cross section, $v_{e} \sigma_{\mathrm{tr}}$, as a function of the kinetic energy $E$. Calculations have been performed for argon, krypton and xenon ions in different ion charge states $Z$. The influence of the surrounding plasma medium on the electron-ion interaction (7) is small in the considered region of mean electron energies. The ion number density in the expression of the screening parameter $\kappa$ is fixed to the initial densities for which we adopt the respective liquid densities of argon $\left(n_{i}=0.00313\right.$ a.u.) krypton $\left(n_{i}=0.00229\right.$ a.u. $)$ and xenon $\left(n_{i}=0.00239\right.$ a.u. $)$.

Classical transport cross sections for electron-ion scattering were calculated for the Herman-Skillman-like potential of Rogers (7). Results are shown for argon and xenon ions of different charge states in figures 2 and 3 as function of the mean electron energy. The values for xenon ions are greater than those of argon ions of the same charge number 


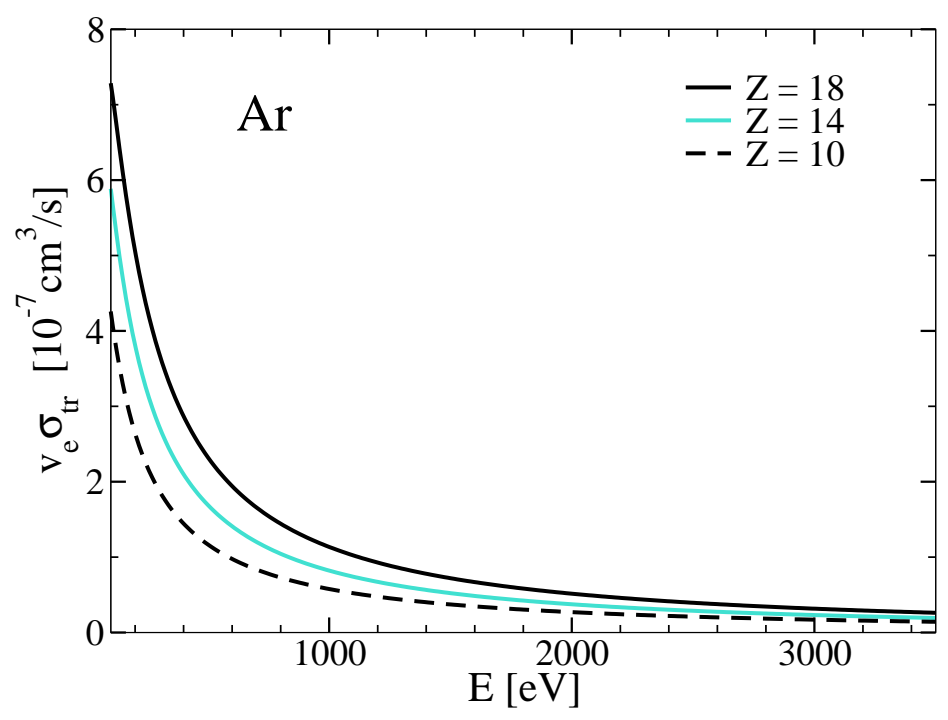

Figure 2. Transport cross sections (multiplied with the velocity) for the model potential (7) as a function of the kinetic energy for argon ions $\mathrm{Ar}^{Z+}$ with charge numbers $Z=10,14,18$.

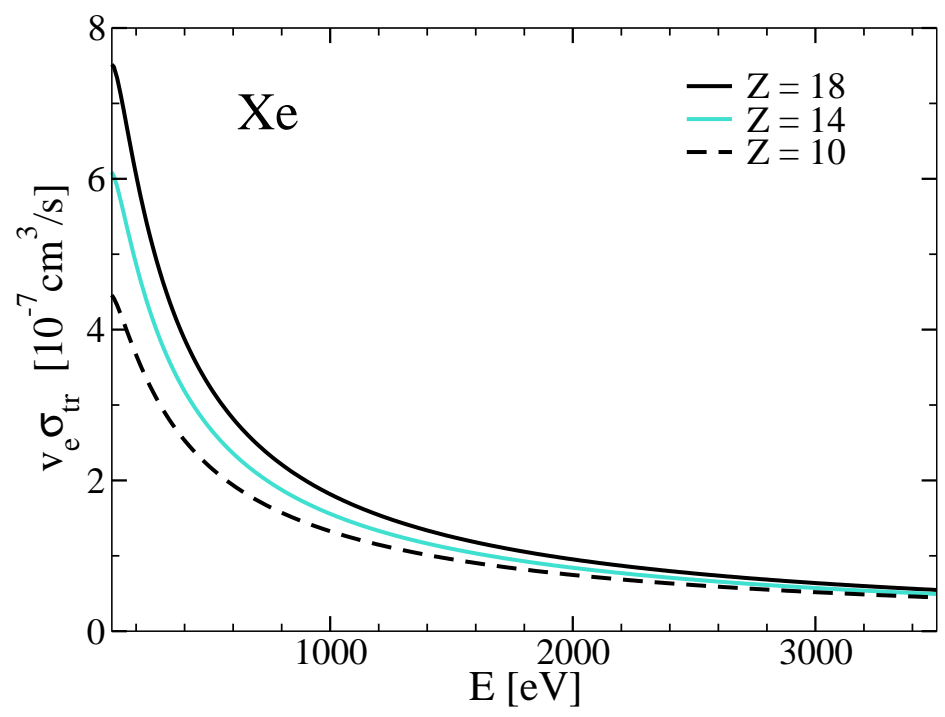

Figure 3. Transport cross sections (multiplied with the velocity) for the model potential (7) as a function of the kinetic energy for xenon ions $\mathrm{Xe}^{Z+}$ with charge numbers $Z=10,14,18$.

which is due to the larger nuclear charge screened by the core electrons. Especially for higher energies, the core region contributes to the transport cross section. The influence of the inner structure is stronger for larger $Z_{\text {nucl }}$ and for lower $Z$. Therefore the values for krypton ions not shown here are in between the Ar and Xe results. We want to note that calculations performed with the simpler Santra-Green potential $V_{S G}$, Eq. (5), including a Debye screening factor give slightly changed results with relative deviations of $\sim 20 \%$. The ratio of the cross sections for the two potentials is of the order of unity over the whole energy range for all charge states between $Z=1$ and $Z=18$. 
It is an important question how different models do compare with each other. We concentrate here on the comparison of collision frequencies basing on classical transport cross sections with those basing on the first Born approximation. Wang et al. [27], e.g., have applied the Born approximation to calculate total cross sections for inverse Bremsstrahlung with effective electron-ion potentials similar to Eq. (5). In the present paper, we calculated classical transport cross sections for the screened model potential $V_{R}^{s c}$ of Rogers given by Eq. (7) and for the Debye potential $V_{D}$. Additionally, we have considered the Born approximation for the model potential $V_{R}^{s c}$.

In Figs. 4, 5 and 6, results are presented for different charge states, $Z$, of xenon atomic ions. The electron number density is adopted to be $n_{e}=Z n_{i}$ with $n_{i}=0.00239$ a.u. Further, in Fig. 5, results for xenon and argon ions are compared for the same charge state, $Z=8$. The collision frequencies are shown for energy ranges in which the respective ion charge states are populated. The overall picture is the following: in the relevant range of mean energy, the collision frequencies for the realistic model potential $V_{R}^{s c}$ (dark full lines) are greater than those for the Debye potential $V_{D}$ (bright full lines). This is the effect of the structure of the ion core, namely the contribution of scattering events with small scattering parameters. Consequently, the differences increase with increasing mean energy (temperature). The influence of the core structure can be seen also in Fig. 5: the collision frequency for xenon ions is higher than that for argon ions of the same charge state. The Born approximation - which was shown in section 4 to be not applicable - overestimates the collision frequency considerably (dashed lines).

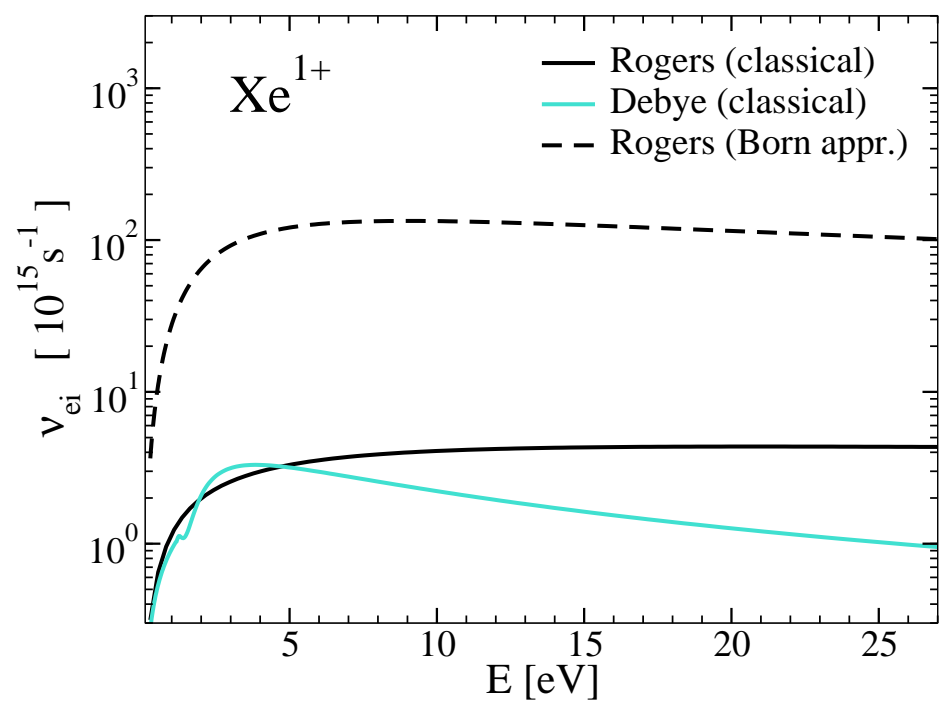

Figure 4. Collision frequency $\nu_{e i}$ for $\mathrm{Xe}^{1+}$ ions as function of the kinetic energy $E=3 / 2 k_{B} T$ calculated for $n_{i}=0.00239$ a.u. in different models, see text. 

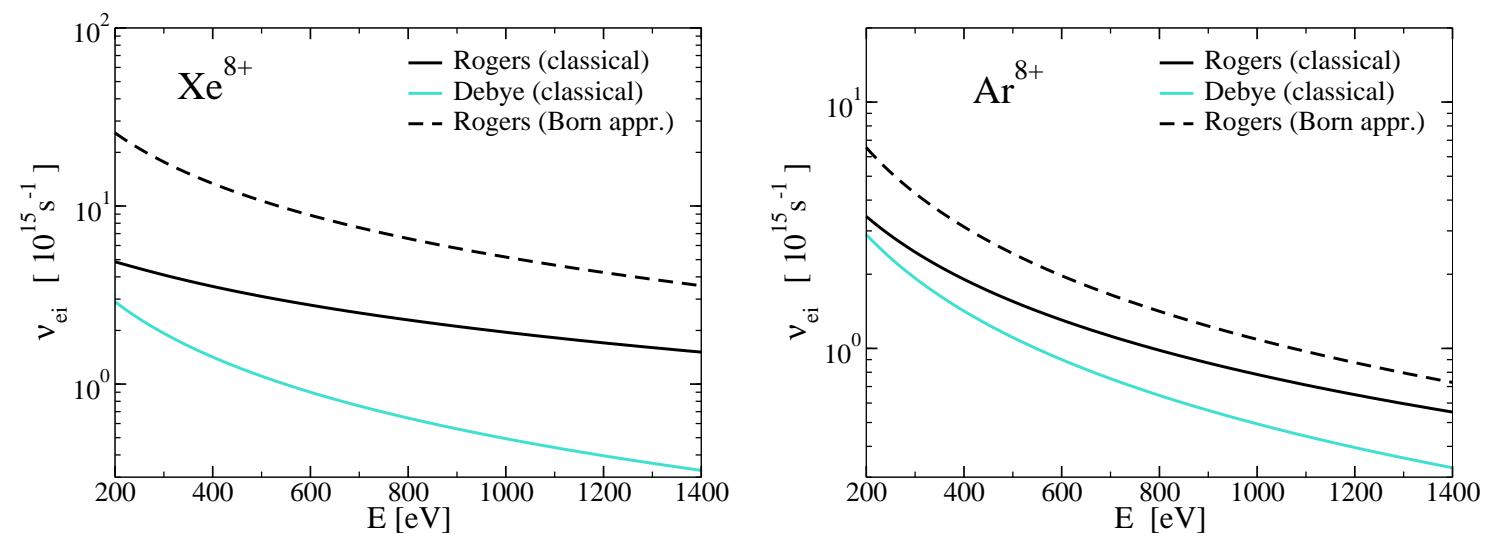

Figure 5. Collision frequency $\nu_{e i}$ for $\mathrm{Xe}^{8+}$ and $\mathrm{Ar}^{8+}$ ions as function of the kinetic energy $E=3 / 2 k_{B} T$ calculated for $n_{i}=0.00239$ a.u. in different models, see text.

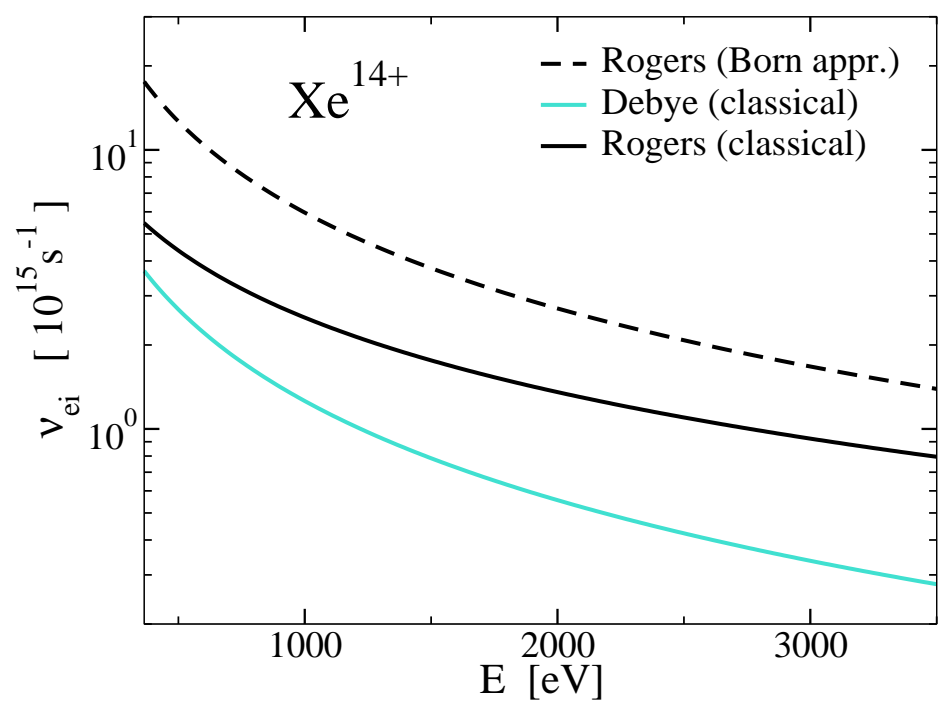

Figure 6. Collision frequency $\nu_{e i}$ for $\mathrm{Xe}^{14+}$ ions as function of the kinetic energy $E=3 / 2 k_{B} T$ calculated for $n_{i}=0.00239$ a.u. in different models, see text.

\section{Conclusion}

Electron-ion collisions were investigated for plasma conditions occuring in atomic noble gas clusters irradiated by femtosecond laser pulses. With increasing electron velocity it becomes important to account for the inner structure of atomic ions having many core electrons. Considering the electron-ion collision frequency, scattering events at the mean electron velocity give the largest contribution. One has to account for the interplay between mean kinetic energy (electron temperature) and ionization balance. The ionization degree in the cluster nanoplasma increases with increasing mean electron kinetic energy.

In a wide parameter range met in typical experiments, the Born approximation is not applicable. We calculated the electron-ion collision frequency on the basis of classical momentum transport cross sections using realistic model potentials. A comparison of 
these results to those for the scattering on Coulomb particles with the same charge shows an enhancement of the collision frequency. Results have been presented especially for xenon ions in different charge states. Although the ions of krypton and argon behave quite similar, for the ions of similar charge states which have more core electrons, the differences are higher.

The differences between the two different model potentials, the Hermann-Skillman type potential of Rogers and the simpler Santra-Green potential, were found to be relatively small. As the additional expenses in the numerical calculations are small, the potential $V_{R}^{s c}$ of Rogers is preferable anyway.

The influence of the laser field on the scattering processes could be neglected for typical laser intensities of approximately $10^{15} \mathrm{~W} / \mathrm{cm}^{2}$. For higher intensities, staying in the non-relativistic regime, one has to generalize the approach of Fraiman et al. [28], see also [29], who considered the scattering on Coulomb centers in external fields, for the case of realistic model potentials.

\section{Acknowledgments}

This work was supported by the Deutsche Forschungsgemeinschaft within Sonderforschungsbereich 652 .

\section{References}

[1] Jha J and Krishnamurthy M 2008 J. Phys. B 41041002

[2] Petrov G M and Davis J 2008 Phys. Plasmas 15056705

[3] Last I and Jortner J 2004 J. Chem. Phys. 1201348

[4] Heidenreich A, Last I, and Jortner J 2008 Eur. Phys. J. D 46195

[5] Islam M R and Saalmann U 2009 J. Phys. B 42

[6] Prigent C, Deiss C, Lamour E, Rozet J-P, Vernhet D, and Burgdörfer J 2008 Phys. Rev. A 78 053201

[7] Taguchi T, Antonsen Jr. T M, Palastro J, Milchberg H and Mima K 2010 Optics Express 182389

[8] Krainov V P and Smirnov M B 2002 Physics Reports 370237

[9] Saalmann U, Siedschlag C H, and Rost J-M 2006 J. Phys. B 39 R39

[10] Krainov V P 2000 J. Phys. B 331585

[11] Dawson J M and Oberman C 1963 Phys. Fluids 6394

[12] Silin V P 1964 Zh. Eksp. Teor. Fiz. 472254 [1965 Sov. Phys.-JETP 20 1510]

[13] Decker C D, Mori W B, Dawson J M, and Katsouleas T 1994 Phys. Plasmas 14043

[14] Mulser P and Schneider R 2008 J. Phys. A: Math. Theor. 42214058

[15] Kroll N M and Watson K M 1973 Phys. Rev. A 8804

[16] Kull H-J and Plagne L 2001 Phys. Plasmas 85244

[17] Bornath Th, Schlanges M, Hilse P, and Kremp D 2001 Phys. Rev E 6426414

[18] Hazak G, Metzler N, Klapisch M, and Gardner J 2002 Phys. Plasmas 9345

[19] Reinholz H, Redmer R, Röpke R, and Wierling A 2000 Phys. Rev. E 625648

[20] Reinholz H and Bornath Th 2008 In: Th. Brabec, editor, Strong Field Laser Physics (Springer Series in Optical Sciences, Vol. 134) (Berlin: Springer-Verlag) 455

[21] Herman F and Skillman S 1963 Atomic Structure Calculations (Englewood Cliffs, New Jersey: Prentice-Hall, Inc.)

[22] Rogers F J 1981 Phys. Rev. A 231008 
Electron-ion collision rates in atomic clusters irradiated by femtosecond laser pulses 12

[23] Santra R and Greene C H 2003 Phys. Rev. Lett. 91233401

[24] Lide D R 1998 Handbook of Chemistry and Physics (Ann Arbor: CRC Press)

[25] Messiah A 1999 Quantum Mechanics (Mineola, N.Y.: Dover Publications, Inc.) Section XIX-7

[26] Newton R G 1982 Scattering Theory of Waves and Particles (Berlin: Springer-Verlag)

[27] Wang F, Weckert F, and Ziaja B 2009 J. Plasma Physics 75289

[28] Fraiman G M, Mironov V A, and Balakin A A 1999 Phys. Rev. Lett. 82319

[29] Brantov A, Rozmus W, Sydora R, Capjack C E, Bychenkov V Yu, and Tikhonchuk V T 2003 Phys. Plasmas 103385 